\title{
EL SIGNIFICADO DEMOGRÁFICO DE LA EDAD Y EL SEXO*
}

\author{
NATHAN KEYFITZ \\ Universidad de California, Berkeley
}

DE Los NIÑos de 15 años de edad, el doble de los huérfanos de madre son huérfanos de padre. En los Estados Unidos existen alrededor de dos abuelas por cada abuelo, y diez tatarabuelas por cada tatarabuelo.

En los Estados Unidos la proporción de personas de más de 65 años es cuatro veces la de Venezuela. ¿Qué tanto de la diferencia se debe a las tasas mayores de natalidad de Venezuela? ¿Qué tanto a las tasas de mortalidad mayores? La teoría que dé respuesta a esto debe también poder mostrar cómo un censo o una muestra en la que sólo aparezca el porciento de población menor de 20 años de edad es suficiente para hacer una buena estimación de su tasa de crecimiento. La teoría de poblaciones estables que sirve para éstos y otros propósitos fue presentada por el versátil Leonhard Euler en 1760, y al igual que muchos descubrimientos matemáticos ha encontrado sus aplicaciones principales dos siglos después. El reconocimiento de la inestabilidad y su modo de operación es mucho más reciente — por ejemplo, en los Estados Unidos el "auge de bebés" de los años cincuenta tiene una serie de repercusiones que podría continuar hasta el siglo xxI. Una de las consecuencias de la abundancia de niños, o de cualquier otra fluctuación similar, es que durante la fase ascendente las jóvenes de 20 a 25 años se enfrentan a una escasez de hombres casaderos, y durante el descenso, unos cuantos años después, los hombres no pueden encontrar parejas de edad adecuada. El estudio de las poblaciones estables y luego de las inestables será la primera tarea de este trabajo.

Estos puntos diversos ayudarán a poner en claro el significado demográfico de la• edad y el sexo. El mejor lugar para empezar a tratarlos se encuentra en el modelo estable unisexual. Inclusive en el modelo unisexual simple, pero aplicado en forma simultánea a hombres y mujeres, compararé las cantidades de ambos sexos, y en especial sus valores relativos en las distintas edades. Otra manera de comparar los

* Investigación patrocinada por un donativo del NSF-62995 mediante contexto de investigación NIH-69-2200 y donativos para enseñanza hechos al Departamento de Demografía, Universidad de California en Berkeley por el Instituto Nacional de Ciencias Médicas Generales (S T01 GMO 1240) y la Fundación Ford. El Dr. Joel E. Cohen llamó mi atención sobre varios errores en una versión anterior. El título del original es "The Demographic Meaning of Age and Sex". La traducción al español es de A. García Rocha. 
sexos consiste en ver cuántos parientes masculinos y cuántos de sexo femenino tiene una persona. En la sección final veremos qué puede decirse acerca de un modelo bisexual genuino que tome en cuenta la edad -aunque algunos problemas matemáticos formidables dificultan las conclusiones precisas.

\section{LA ESTABILIDAD EN EL MODELO UNISEXUAL}

Para empezar con la parte más accesible matemáticamente, pensemos en la distribución por edades de las mujeres de una población en la que las tasas de mortalidad y natalidad han permanecido constantes durante mucho tiempo. El análisis no puede quedar mejor acomodado que como lo hizo Euler en 1760 en un trabajo poco conocido.

Euler se interesó por una gran variedad de aplicaciones de las matemáticas. Profundamente internacional, nacido en Suiza, Euler pasó parte de sus años más productivos como matemático de la corte de Federico el Grande, y murió en San Petersburgo en 1783. Representa el espíritu de la Ilustración de devoción a la ciencia, del cual han surgido sociedades nacionales tales como la Asociación Norteamericana para el Avance de la Ciencia (AAAS).

La contribución de Euler a la teoría de la población fue desarrollada durante su estancia en Berlín, y la dio a conocer en las conferencias de 1760 de la Real Academia Belga de Ciencia y Literatura. Me refiero a lo anterior en esta parte de mi exposición no sólo porque Euler inventó el tema, sino porque nadie hasta ahora lo ha expuesto en forma más clara. (Suprimiré, sin embargo, su francés anticuado y su no menos anticuada notación.)

Son suficientes dos principios - dice Euler- para desarrollar una teoría de las distribuciones por edades. El primero es el de mortalidad fija, en el cual, de una cohorte de $B$ nacimientos, el número de sobrevivientes $x$ años después puede escribirse $B \ell_{x}$. Con base en el conjunto de números $\ell_{v}$ podemos encontrar la esperanza de vida de un individuo, el valor de una renta anual de su propiedad, y muchos otros asuntos concernientes a los individuos.

Pero, a diferencia de los demógrafos modernos, el interés de Euler no se centraba en los valores individuales esperados. Inmediatamente pasa a tratar el problema en relación con poblaciones, y por ello necesita un segundo supuesto, que él llama multiplicación. Ésta fue la ley de crecimiento geométrico que Malthus empleó 40 años después; dice simplemente que si el número de nacimientos excede al de defunciones, entonces la población crecerá, y si el cociente entre el crecimiento y la población es constante, entonces tanto la po. blación total como los nacimientos crecerán geométricamente.

Estos supuestos de mortalidad y crecimiento fijos son todo lo que se necesita para formular el modelo estable, incluida la determinación de la distribución por edades. Porque, si los nacimientos de este año son $B$, los nacimientos del año próximo serán $e^{r} B$, donde $e^{r}$ es la tasa de crecimiento supuesta fija; los nacimientos de los años siguientes serán $e^{2 r} B$, etc. Pensemos ahora en el futuro, hacia la fecha en la que habrán muerto todos los que han nacido hasta ahora, digamos dentro 
de 100 años. Entonces la población consistiría en los nacimientos de este año multiplicados por la probabilidad de vivir hasta los 100 años, que es $B \ell_{100}$, más los nacimientos de años siguientes, $B e^{r}$, multiplicados por $\ell_{9 g}$, es decir $B e^{r} \ell_{99}$; los nacimientos del año siguiente multiplicados por $\ell_{98}$, es decir $B e^{r} \ell_{98}$, etc.; terminando con los nacimientos dentro de 100 años, $B e^{100 r}$. Sumando todo para los 100 años tendremos como población total de esa fecha

$$
B \boldsymbol{R}_{100}+B e^{r} \boldsymbol{R}_{99}+B e^{2 r} \boldsymbol{\beta}_{98}+\ldots+B e^{100 r},
$$

donde el primer término representa las personas de 100 años de edad, el segundo las de 99 , etc. Poniendo $B e^{100 r}$ como factor e invirtiendo el orden de los términos, resulta la población total de esa fecha, diga$\operatorname{mos} P_{100}$ :

$$
P_{100}=B e^{100 r}\left(1+8_{1} e^{-r}+8_{2} e^{-2 r}+\ldots+8_{100} e^{-100 r}\right)
$$

donde el primer término se refiere a la edad 0 , el segundo a la edad 1 , etcétera.

Al dividir ambos lados de la ecuación por la población $P_{100}$, y recordando que la relación entre los nacimientos y la población ha de ser constante, según la hipótesis de multiplicación, digamos $b$, tenemos entonces la identidad

$$
1=b+b 8_{1} e^{-r}+b 8_{2} e^{-2 r}+\ldots
$$

Los distintos términos del lado derecho se refieren a las distintas edades de la población a partir de cero, y en vista de que suman 1, en esta forma cada término debe ser una proporción del total. Hemos seguido a Euler a lo largo de su descubrimiento de la distribución estable por edades, incluyendo su demostración de que depende sólo de la tabla de vida y de la tasa global de crecimiento de la población. Al suponer fijos la tabla de vida y el crecimiento, la distribución por edades queda completamente determinada. Un tratamiento moderno sería más refinado sólo en el detalle de especificar la mortalidad infantil, desde el nacimiento hasta el final del año cuando se estima la distribución por edades.

Podemos eludir cualquier complicación que surja del hecho de que los nacimientos acontecen a todo lo largo de cada año, llevando la fórmula de Euler a términos continuos. Si la proporción buscada de la población entre las edades $a$ y $a+d a$ es $c(a) d a$, entonces tenemos:

$$
c(a) d a=b e^{-r a} R_{a} d a,
$$

que se obtiene simplemente reduciendo a $d a$ el intervalo de tiempo de un año empleado por Euler.

Éste señaló que uno de los usos de este resultado es que si conocemos la distribución por edades, por ejemplo de un censo, y conocemos la tasa de natalidad y la de crecimiento natural, entonces podemos inferir la tabla de vida:

$$
8_{a}=\frac{c(a) e^{r a}}{b}
$$


que resulta directamente de la expresión anterior referente a $c(a) d a$.

Este resultado de Euler ha tenido aplicación considerable en años recientes (Arriaga, 1968, y otros). En muchos países existen censos, pero en ellos no se han establecido sistemas de registro de defunciones exactos, y por ello la expresión de Euler sobre la distribución por edades es una herramienta poderosa, aplicable siempre que puedan suponerse tasas aproximadamente constantes.

\section{Aplicación a inferencias a partir de datos incompletos}

Euler concluyó sus investigaciones sobre la teoría de las poblaciones estables en este punto más o menos, y el siguiente desarrollo sobre la línea de nuestro interés presente hubo de esperar 200 años hasta que Ansley Coale observó que si conocemos la tabla de vida $\ell_{x}$ y la proporción $\alpha$ de individuos menores de 25 años, por ejemplo, podemos entonces determinar univocamente $r$, la tasa de crecimiento de la población. A partir de la tasa fija de crecimiento (desconocida), y de la tabla de vida $\ell_{x}$ y la proporción a menor de 25 años, tenemos la ecuación

$$
\alpha=\frac{\int_{0}^{25} e^{-r x} 8_{x} d x}{\int_{0}^{100} e^{-r x} 8_{x} d x}
$$

Resolverla para $r$ es difícil si requerimos una forma cerrada, es decir, si deseamos expresar $r$ como una función general de $\alpha$ y las $\ell_{x}$. En vista de que la ecuación es común a muchos temas de la demografía matemática, encontraremos instructivo considerar cómo puede ser resuelta.

Una manera consiste en construir tablas de distribuciones estables por edades para distintos $\ell_{x}$ y $r$. Éste fue el método utilizado por Coale y Demeny (1966). Uno busca en el libro de tablas aquella que tiene las mismas $\alpha$ y $g_{v}$ que el problema, y observa el valor de $r$ que aparece. El método puede hacerse tan preciso como sea necesario mediante tablas bien graduadas que luego pueden interpolarse.

Un método alternativo es por aproximaciones sucesivas mediante una computadora. Sacamos de la integral del numerador anterior el factor $e^{- \text {ior }}$, de modo que quede en la forma

$$
e^{-10 r} \int_{0}^{25} e^{-r(x-10)} 8_{v} d x
$$

que tiene valor idéntico. Del mismo modo, extraemos del denominador el factor $e^{-25 r}$, y de nuevo multiplicamos dentro de la integral de manera de no cambiar nada. La combinación de estos dos factores nos da $e^{15 r}$ fuera del cociente de integrales. Después de dividir ambos 
lados por el cociente de integrales, tomar logaritmos y dividir por 15, tenemos

$$
r=\frac{1}{15} \ln \frac{\alpha \int_{0}^{100} e^{-r(x-25)} \beta_{x} d x}{\int_{0}^{25} e^{-\tau(x-10)} \lambda_{x} d x}
$$

Esta es exactamente la misma ecuación anterior, ya que cada cambio efectuado opera exactamente de la misma manera en ambos lados de la ecuación. De este modo hemos encontrado una solución para $r$. Tómese un valor de $r$ arbitrario al lado derecho; con éste y las $\boldsymbol{B}_{x}$ conocidas evalúense ambas integrales, multiplíquese por a y tómese el logaritmo y obténgase así un valor de $r$ mejorado, digamos $r^{*}$. Insertando éste en el lado derecho nos permite mejorar $r$ aún más. Después de unas cuatro reiteraciones ya no se presentan cambios en $r, \mathrm{y}$ el proceso puede suspenderse. Cuando el proceso converge sabemos que debe hacerlo hacia la respuesta correcta; ya que la segunda forma de la ecuación es en realidad equivalente a la primera, cualquier $r$ que satisfaga una debe hacer otro tanto con la otra. Entonces, en ésta y en muchas otras ecuaciones, todo lo que hacemos es manipularlas hacia una forma cuyo lado derecho sea relativamente insensible al valor de la incógnita, luego escogemos un valor arbitrario de ésta y llevamos a cabo el proceso hasta que ya no se presente cambio alguno. Es posible derivar un número infinito de formas, y mientras se presente una convergencia razonablemente rápida no es necesario ser muy selectivos respecto de cuál utilizar.

Como ejemplo, considérese la información de Colombia en 1965. La solución de la ecuación anterior, con $\alpha=0.643$ y $\ell_{x}$ tomadas de una tabla de vida, proporciona el valor de $r=0.0333$. Todo esto utilizando sólo la distribución por edades de las mujeres. El mismo argumento puede aplicarse a los hombres, utilizando la proporción de hombres menores de 25 años y la tabla de vida masculina. La estimación de $r$ obtenida de este modo es 0.0329 . Cuando consultamos las estadísticas vitales registradas encontramos una tasa de natalidad de $\mathbf{0 . 0 3 8 4}$ y una tasa de mortalidad de 0.0099 , que llevan a una tasa de crecimiento natural de 0.0285 .

Nuestro cálculo a partir del censo dio una tasa de crecimiento de 0.0333 para las mujeres y 0.0329 para los hombres, mientras que los nacimientos menos las defunciones dan la cifra mucho menor de 0.0285 para ambos sexos. ¿Cuál habremos de creer? Se dispone de mayor evidencia, y uno puede con certeza apostar que la tasa de crecimiento natural real es mucho más parecida a nuestra estimación, digamos $3.3 \%$, que a las estadísticas vitales oficiales.

Muchas otras ecuaciones surgen en esa parte nueva de la demografía en donde se hace inferencia con base en información incompleta. El campo ha sido desarrollado por Brass, Coale, Demeny, Henry, López y otros.

Pero el interés de este trabajo no es tanto el de llenar la informa- 
ción incompleta e inconsistente sino el de interpretar la información buena. Para este propósito la distribución estable por edades de Euler es especialmente útil. Procedemos a aplicarla a cada sexo, antes de hacer nada con el modelo de dos sexos.

\section{EL MODELO ESTABLE Y LA REALIDAD}

Hemos probado en forma indirecta el modelo de Euler mostrando que da una estimación razonable de la tasa de crecimiento natural, pero es deseable hacer una prueba más directa. Esto se hace para comparar la distribución de la población observada en un censo con lo que sería según la fórmula de Euler.

Se ha hecho esta comparación para unas 600 poblaciones, y el mejor grado de semejanza en todas las fechas y lugares parece ser el del censo de Inglaterra y Gales en 1881. En esa fecha las distribuciones porcentuales de las mujeres de las poblaciones observada y estable fueron muy parecidas.

\begin{tabular}{ccc}
\hline Observada & Estable & $\begin{array}{c}\text { Diferencia } \\
\text { observada-estable }\end{array}$ \\
\hline 13.2 & 13.1 & 0.1 \\
11.8 & 11.4 & 0.4 \\
10.5 & 10.4 & 0.1 \\
9.6 & 9.5 & 0.1 \\
9.1 & 8.6 & -0.5 \\
$\ldots$ & $\ldots$ & $\ldots$ \\
\hline
\end{tabular}

Una medida de la diferencia entre la observada y la estable es el índice de disimilitud $\Delta$-que es la suma de las diferencias porcentuales positivas. Para Inglaterra y Gales de 1881 el índice fue $\Delta=1.4$, aparentemente el menor de todos en cualquier fecha y lugar. Los Estados Unidos en 1967 mostraron un valor mucho mayor: $\Delta=6.3 \mathrm{La}$ mayor distancia en un país grande es la de Japón en 1966 con $\Delta=26.3$. $\mathrm{La}$ inestabilidad de Europa según el índice $\Delta$ es aproximadamente igual a la de Estados Unidos. La mayoría de los países subdesarrollados de la actualidad muestran valores de $\Delta$ entre 3 y 5 .

Para muchos propósitos de análisis el modelo es indispensable, y el modelo estable es especialmente conveniente: de aquí nuestro interés por conocer qué tanto explica. Sin embargo, sólo hasta cierto punto podemos confiar en sus resultados. Por ejemplo, para comparar Venezuela con los Estados Unidos en cuanto a la proporción de personas menores de 15 años, notamos que los porcientos observados, 45.4 y 30.9 , en 1965, representan un exceso de Venezuela de 14.5 puntos porcentuales. Si hacemos la misma comparación mediante el modelo estable encontramos 46.5 contra 28.4, lo cual da un exceso en Venezuela de 18.1 puntos. La respuesta del modelo estable tiene el sentido correcto pero es demasiado elevada: $25 \%$. Podemos tener 
precaución por el hecho de que, para los Estados Unidos, $\Delta$ fue superior a 6. Parece prudente usar el modelo estable principalmente para poblaciones con $\Delta$ baja, aunque aún en las otras el modelo nos dice algo.

\section{EXPLICACIÓN DE DIFERENCIAS EN LAS PROPORCIONES}

Como segunda aplicación de la población estable de Euler, consideramos el hecho de que en Venezuela el $45.4 \%$ de la población es menor de 15 años. Esta elevada dependencia es un obstáculo al desarrollo; los gastos se dirigen a la crianza de niños que en otras circunstancias serían para construir fábricas. En vista de que los recursos son finitos, el país puede elegir entre tener pocos niños con buenas perspectivas de empleo productivo o muchos para los cuales no habrá equipo para emplearlos cuando lleguen a la edad de trabajar.

Tales consideraciones nos motivan a hacer comparaciones con otros países en la época en que se encontraban en una etapa correspondiente de crecimiento económico. Observamos, por ejemplo, que en Suecia, alrededor de 1805 , sólo el $32.7 \%$ de su población era menor de 15 años. La carga de dependencia - es decir, el cociente de los menores de 15 y mayores de 65 sobre los del grupo de edades 15-65- fue de 0.60 para Suecia y de 0.95 para Venezuela. Las personas en edad de trabajar tuvieron también en promedio más de un $50 \%$ más de dependientes en Venezuela que en Suecia. La menor proporción de niños en Suecia le dio ventaja sustancial sobre Venezuela en cuanto a las posibilidades de inversión industrial.

Nuestra interrogante es el grado en que la diferencia general entre Suecia y Venezuela se debe a diferencias entre sus tasas de mortalidad. Para dar respuesta a tal cuestión necesitamos un modelo en el que podamos hacer variar primero los nacimientos y luego las defunciones; sin un modelo sólo podemos observar que Suecia tuvo una tasa de mortalidad mucho mayor que la de Venezuela -24.9 por mil contra 7.1 por mil-y que por otra parte Venezuela tiene una tasa de natalidad mayor que la que tenía Suecia - 43.5 contra 31.3 , y esto concluye el asunto. Afortunadamente encontramos que la $\Delta$ entre las edades de población estable y población observada en Suecia es tan baja como 1.6 en 1805, y 3.4 para Venezuela en 1965. Estos valores bajos alientan a proseguir utilizando las fórmulas de Euler. No necesitamos buscar más un modelo que nos permita efectuar un experimento: para nivestigar específicamente qué proporción sería menor de 15 años en un país con las tasas de mortalidad de Suecia y las de natalidad de Venezuela. Mediante la comparación de este país hipotético con la Suecia actual obtenemos un indicador puro del efecto de la fecundidad cuando la mortalidad es constante.

El porciento menor de 15 años con las tasas de mortalidad y natalidad suecas fue 31.3. La proporción correspondiente con las tasas de mortalidad de Suecia y las de natalidad de Venezuela fue 43.6. Por ello el efecto de la fecundidad venezolana es elevar la proporción de menores de 15 en 12.3 puntos. Estas cifras aparecen en la primera sección del cuadro 1, acomodadas para mostrar los efectos de todas 
las combinaciones de mortalidad y fecundidad entre cinco países. La mortalidad de cada celda es la de la población en la parte superior de la columna; la fecundidad es la de la población a la izquierda de cada renglón. Las cifras de la diagonal son las normales de cada población, correspondientes a sus propias mortalidad y fecundidad.

\section{Cuadro 1}

ASPECTOS DE LA DISTRIBUCTÓN POR EDADES Y LAS TASAS DE CRECIMIENTO OBTENIDAS MEDIANTE LA COMBINACIÓN DE TASAS DE MORTALIDAD Y NATALIDAD FEMENINA DE Cinco PaÍses: Venezuela, 1965; los Estados Unidos, 1963; Madagascar, 1966; INGLATERRA Y GALES, 1968; SUECIA, 1803-1807

\begin{tabular}{|c|c|c|c|c|c|c|c|}
\hline & & \multicolumn{6}{|c|}{ Tagas de natalidad por edades do: } \\
\hline & & Vonozuein & $\begin{array}{l}\text { Eatudor } \\
\text { Unido: }\end{array}$ & Madagascar & Inglaterra & & Snecie \\
\hline \multicolumn{8}{|c|}{ Proporcion de menores de 15 miños } \\
\hline \multirow{4}{*}{$\begin{array}{l}8 \\
8 \\
8 \\
8 \\
8 \\
8 \\
8 \\
8 \\
8\end{array}$} & $\begin{array}{l}\text { Vonezuela } \\
\text { Estados Unidos } \\
\text { Kadagascar } \\
\text { Inglaterra } \\
\text { Sueola }\end{array}$ & $\begin{array}{l}47.7 \\
48.5 \\
45.0 \\
48.5 \\
43.6\end{array}$ & $\begin{array}{l}23.9 \\
24.5 \\
22.0 \\
24.5 \\
21.0\end{array}$ & $\begin{array}{l}47.8 \\
48.6 \\
45.2 \\
48.6 \\
43.8\end{array}$ & $\begin{array}{l}23.6 \\
24.2 \\
21.8 \\
24.2 \\
20.8\end{array}$ & $\therefore$ & $\begin{array}{l}34.2 \\
34.8 \\
32.1 \\
34.8 \\
31.3\end{array}$ \\
\hline & \multicolumn{7}{|c|}{ Cocientes de dependencia (porciento) } \\
\hline & $\begin{array}{l}\text { Venezuela } \\
\text { Estados Unidos } \\
\text { Madagascar } \\
\text { Inglaterra } \\
\text { Suecia }\end{array}$ & $\begin{array}{r}102.1 \\
105.4 \\
91.3 \\
105.2 \\
85.6\end{array}$ & $\begin{array}{l}58.8 \\
61.1 \\
51.5 \\
60.3 \\
46.7\end{array}$ & $\begin{array}{r}102.4 \\
105.6 \\
91.8 \\
105.5 \\
86.2\end{array}$ & $\begin{array}{l}58.7 \\
60.9 \\
51.3 \\
60.1 \\
46.6\end{array}$ & . & $\begin{array}{l}70.3 \\
72.5 \\
62.8 \\
72.1 \\
58.9\end{array}$ \\
\hline & \multicolumn{7}{|c|}{ Proporcion de personas mayores de 65 añon } \\
\hline 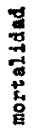 & $\begin{array}{l}\text { Vonezuele } \\
\text { Estados Unidor } \\
\text { Madagasoar } \\
\text { Inelaterra } \\
\text { Suecia }\end{array}$ & $\begin{array}{l}2.8 \\
2.8 \\
2.7 \\
2.8 \\
2.5\end{array}$ & $\begin{array}{l}13.1 \\
13.5 \\
12.0 \\
13.1 \\
10.9\end{array}$ & $\begin{array}{l}2.8 \\
2.8 \\
2.7 \\
2.7 \\
2.5\end{array}$ & $\begin{array}{l}13.3 \\
13.7 \\
12.2 \\
13.3 \\
11.0\end{array}$ & : & $\begin{array}{l}7 \cdot 1 \\
7 \cdot 3 \\
6.5 \\
7 \cdot 1 \\
5.8\end{array}$ \\
\hline 8 & \multicolumn{7}{|c|}{ Edad prowedio } \\
\hline$\underset{*}{\stackrel{8}{*}}$ & $\begin{array}{l}\text { Venezuela } \\
\text { Egtados Unidos } \\
\text { Yadagascar } \\
\text { Inglaterra } \\
\text { Suecia }\end{array}$ & $\begin{array}{l}21.1 \\
20.8 \\
22.0 \\
20.8 \\
22.5\end{array}$ & $\begin{array}{l}35.5 \\
35.4 \\
36.0 \\
35.2 \\
36.1\end{array}$ & $\begin{array}{l}21.0 \\
20.8 \\
21.9 \\
20.8 \\
22.4\end{array}$ & $\begin{array}{l}35.7 \\
35.7 \\
36.2 \\
35.5 \\
36.2\end{array}$ & & $\begin{array}{l}28.3 \\
28.2 \\
29.0 \\
28.1 \\
29.1\end{array}$ \\
\hline \multicolumn{8}{|c|}{ Tasa intrinseca de crecimiento natural $(x)$} \\
\hline & $\begin{array}{l}\text { Venezuela } \\
\text { Estados Unidos } \\
\text { Nadagascar } \\
\text { Inglaterra } \\
\text { Suecia }\end{array}$ & $\begin{array}{l}38.5 \\
40.7 \\
22.3 \\
41.0 \\
24.3\end{array}$ & $\begin{array}{r}4.9 \\
7.1 \\
-11.3 \\
7.4 \\
-9.4\end{array}$ & $\begin{array}{l}38.6 \\
40.8 \\
22.5 \\
41.1 \\
24.6\end{array}$ & $\begin{array}{r}4.5 \\
6.7 \\
-11.6 \\
7.0 \\
-9.6\end{array}$ & & $\begin{array}{r}19.5 \\
21.5 \\
3.8 \\
21.8 \\
6.4\end{array}$ \\
\hline
\end{tabular}

¿Cuál fue el efecto de la mortalidad? Para investigarlo comparamos el $31.3 \%$ de menores de 15 años de Suecia con el que se habría encontrado en un país hipotético con las tasas de natalidad de Suecia y las de mortalidad de Venezuela. Esto resulta ser de $34.2 \%$, sólo 2.9 puntos porcentuales superior al de Suecia.

Esto nos da la respuesta que buscamos: de la diferencia entre el $31.3 \%$ de Suecia y el 47.7 de Venezuela, el 12.3 se debió a diferencias en la fecundidad y 2.9 a diferencias en la mortalidad. Si hacemos el cálculo en la otra dirección - partiendo de Venezuela en lugar de Suecia- encontramos 12.5 y 2.2 , donde la discrepancia se debe a la interacción. Interacción quiere decir que el efecto de los nacimientos 
no es el mismo ante la presencia de la mortalidad de Suecia que ante la de Venezuela, y ello introduce una incertidumbre en la conclusión. Podemos decir que, en el caso que nos ocupa, el efecto de la fecundidad sobre la proporción de menores de 15 años es cuatro o cinco veces tan importante como el efecto de la mortalidad.

Cualquiera otra diferencia entre las edades de las dos poblaciones puede ser analizada de manera similar. El cociente de dependencia se define como 100 veces el total de menores de 15 años y mayores de 65 , dividido por el número de personas entre 15 y 65 ; éste fue $58.9 \%$ en Suecia y 102.1 en Venezuela. Cerca de 29 de la diferencia se debió a la fecundidad y 14 a la mortalidad. Una vez más, la edad promedio de la población fue de 21.1 años en Venezuela y 29.1 años en Suecia. Cerca de 7.2 años de la diferencia se debe a la fecundidad y 1.1 a la mortalidad.

Considérese el tercer grupo de cifras, el de porcientos de personas de 65 y más años. La cifra del extremo superior izquierdo resulta de las tasas de mortalidad y natalidad de Venezuela; la siguiente cifra sobre la diagonal proviene de las tasas de mortalidad y fecundidad de los Estados Unidos. ¿En qué medida la gran diferencia - 2.8\% contra $13.5 \%$ - obedece a la diferencia entre las tasas de natalidad de los dos países, y en qué medida a las tasas de mortalidad? Al comparar a lo largo del primer renglón, que equivale a mantener en ambas poblaciones las tasas de mortalidad de Venezuela pero permitiendo a cada país tener su propia fecundidad, quedan explicadas casi todas las diferencias, lo cual se obtiene también comparando el segundo renglón, en el que se conservan las tasas de mortalidad de los Estados Unidos. Por otra parte, al comparar por columnas, lo cual equivale a mantener fijas las tasas de natalidad, pero conservando las tasas de mortalidad de cada país, encontramos muy poco reflejo de las diferencias globales. Es evidente que la proporción de personas de edades altas en los países difiere porque sus tasas de natalidad difieren, y difícilmente porque sus tablas de vida sean diferentes.

Todo ello se basa en el modelo estable, y constituye la afirmación más exacta que podemos hacer sin acudir a modelos más complejos. De hecho los porcientos de mayores de 65 observados fueron 2.99 para Venezuela y 9.50 para los Estados Unidos, un cociente como de cuatro a uno contra el cinco a uno del modelo estable. Con este grado de aproximación el cuadro 1 permite el análisis de las diferencias observadas; el análisis tendrá mayor valor cuando las poblaciones sean más semejantes al modelo estable.

Pudimos haber pensado que una población sería más vieja -de mayor edad promedio- si tuviera mortalidad más favorable. Aquí, entonces, la sorpresa es doble: la proporción de menores de 15 años, la edad promedio, y otros aspectos, dependen más de las diferencias de natalidad que de las de mortalidad. Más aún, Suecia tuvo mortalidad mucho mayor que Venezuela y sin embargo su población es más vieja. Esta última paradoja surge de diferencias en la incidencia de la mortalidad por edades - la de Suecia fue particularmente seria en las edades jóvenes. 
Poblaciones mantables

La explicación de las diferencias de las distintas edades entre las proporciones observadas y las del modelo puede ser por sí misma instructiva. Se tomará después el censo de 1960 de los Estados Unidos como ejemplo de una distribución por edades que difiere considerablemente de la estable. El valor de $\Delta$ fue 9.6, bastante más elevado que el que encontramos en Europa y en los mismos Estados Unidos en la mayor parte de la historia. La proporción estable de menores de 15 años fue demasiado alta, 12.7 contra el 10.9 observado, debido al descenso de las tasas de natalidad que había empezado alrededor de 1957-1958. Por otra parte, la proporción estable fue baja en las edades $35-39$, 6.0 contra 7.0 observado, lo cual refleja las bajas tasas de natalidad durante los años treinta.

E1 método que resulta para analizar las distribuciones por edades tiene tres etapas. La primera consiste en comparar con las edades estables; ello nos dice qué parte es totalmente explicable en términos de las tasas corrientes de natalidad y mortalidad, sin acudir a factores históricos. La segunda etapa es ver a qué grado las diferencias entre lo observado con relación a lo estable pueden ser atribuidas a tendencias recientes de los nacimientos y las defunciones. La tercera etapa es la de explicar las discrepancias residuales mediante sucesos históricos extraordinarios. Éstos incluyen las guerras, que reducen el número de hombres alrededor de los 20 años de edad; la inmigración, que tiende a elevar el número de hombres de alrededor de 20 años de edad; y las epidemias, que reducen en especial el número de niños pequeños. En esta parte del análisis la teoría no es de mucha ayuda; debemos buscar en los datos para ver lo que ha sucedido.

\section{EXPLICACIÓN DE LA RELACTÓN DE MASCULINIDAD}

Para el análisis de las relaciones de masculinidad se encontrará útil esencialmente el mismo método utilizado en el tratamiento de las distribuciones por edades. Nuestra investigación, como antes, partirá de las tasas de natalidad y mortalidad corrientes por edades, en relación ahora con los dos sexos por separado, con lo cual puede construirse un modelo estable: lo que quede sin explicar con el modelo estable basado en las tasas corrientes será referido a tendencias de las tasas en los años recientes; lo que quede aún sin explicación, será manejado mediante un estudio de la migración y de los cambios repentinos de las tasas de mortalidad debidos a las guerras, epidemias y otros sucesos históricos. El método está implícito en un tra. bajo de Hawley (1950).

Para convertir el modelo unisexual utilizado para el análisis de las edades en un modelo bisexual (muy primitivo) que necesitamos ahora, debemos agregar un elemento de información: la relación de masculinidad al nacimiento, denotada por $s$. Si por cada mujer nacen $s$ varones, y nacen $B$ mujeres en el año en curso, entonces el número de varones es $s B$. Exactamente mediante el mismo argumento usado 
por Euler, podemos escribir ahora la distribución por sexo y por edad si el régimen de mortalidad y fecundidad es fijo, y la población crece en forma geométrica a una tasa de crecimiento $e^{5 r}$ durante un período de cinco años. Se supone que en nuestro modelo estable los nacimientos, al igual que cada grupo de edad y sexo, crecen a esta tasa. Los grupos de edad quinquenales son los que surgen en la práctica. Los sobrevivientes al final del período quinquenal de $B$ nacimientos femeninos por año serán

$$
B \int_{0}^{5} 8_{t} d t
$$

que denotaremos con $B_{5} L_{0}$. Los sobrevivientes al grupo de edad 5-9 serán $B_{5} L_{5}$, etc. Los nacimientos masculinos correspondientes son $s B$, y los sobrevivientes al final del período quinquenal son $s B_{5} L_{0}^{*}$, al final del segundo período quinquenal $s B_{5} L_{5}^{*}$, etc. Esto da la cantidad en cada edad y sexo en el modelo estable ampliado según las expresiones aproximadas siguientes:

\begin{tabular}{rcc}
\hline Edad & Hombres & Mujeres \\
\hline $0-4$ & $s B_{5} L_{0}^{*} e^{-21 / 2 r}$ & $B_{5} L_{0} e^{-21 / 2 r}$ \\
$5-9$ & $s B_{5} L_{5}^{*} e^{-71 / 2 r}$ & $B_{5} L_{5} e^{-71 / 2 r}$ \\
$10-14$ & $\cdots$ & $\cdots \cdots$ \\
$15-19$ & $\cdots$ & $\cdots$ \\
$\cdots \cdots$ & $\cdots$ & \\
\hline
\end{tabular}

Si por cualquier razón se hace necesario interpretar éstos en términos absolutos en lugar de proporciones, podemos decir que son los números que se encontrarían en una población en la cual los nacimientos anuales masculinos son $s B$ y los femeninos $B$. Al dividir por $B$ se obtiene la distribución por eđad y sexo por nacimiento femenino.

A partir de estas proporciones, la relación de masculinidad para cualquier edad está dada por el cociente de la cifra correspondiente a hombres y la correspondiente a mujeres. Si $r$ es la misma en ambos sexos, como debe ser a largo plazo, entonces la exponencial de la relación de masculinidad se cancela, y ésta, para la edad $x$ a $x+4$ es $s L_{x}^{*} / L_{x}$. Nuestra primera conclusión es que la relación de masculinidad en cualquier edad depende sólo de las dos tablas de vida y de la relación de masculinidad al nacimiento. No depende en absoluto de la tasa de crecimiento $r$ ni de la natalidad. Al sustituir las cifras de 1965 de los Estados Unidos para las edades 70-74 el resultado es

$$
s L_{70}^{*} / L_{70}=1.0472 \frac{224489}{326260}=0.7205
$$




\section{Gráfica 1}

COCIENTE ENTRE LA MORTALIDAD MASCULINA Y FEMENINA POR EDAD;

EsTadOS UNIDOS, 1967; EUROPA, 1965

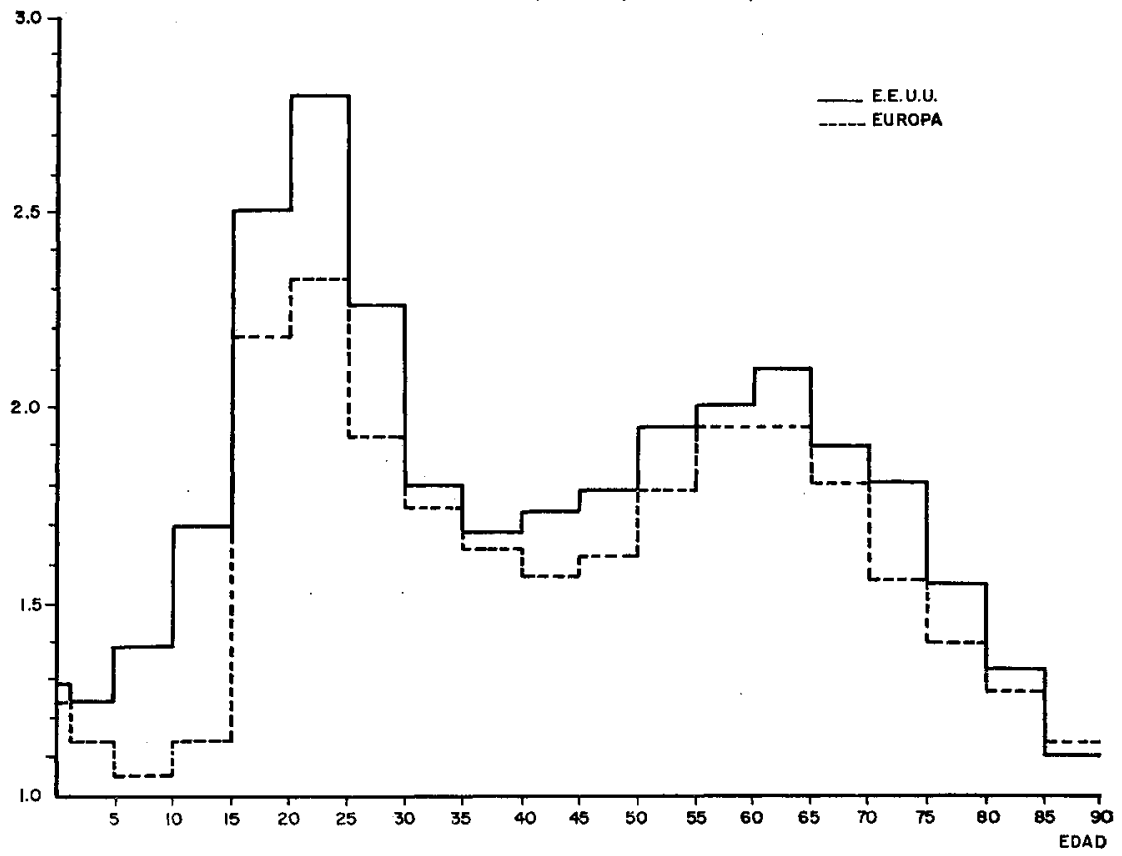

Éste ha de compararse con la relación de masculinidad observada en 1965 en los Estados Unidos que resultó ser 0.7856. En el cuadro 1-a aparecen comparaciones similares para otras edades.

Cuadro 1 a

Estados UNIDOS: CoMparación DE LAS RELACIONES DE MASCULINIDAD OBSERVADAS Y ESTABLES, 1965

\begin{tabular}{|c|c|c|c|c|c|c|}
\hline \multirow{3}{*}{ Edad: } & \multicolumn{3}{|c|}{ Observade } & \multirow{2}{*}{\multicolumn{2}{|c|}{$\begin{array}{r}\text { Calculada oon base en } \\
\text { de estabilida } \\
\text { r }=1.0472\end{array}$}} & \multirow{2}{*}{ supuesto } \\
\hline & \multirow{2}{*}{$\begin{array}{l}\text { Uombros } \\
\text { (miles) }\end{array}$} & \multirow{2}{*}{$\begin{array}{l}\text { Mu jeros } \\
\text { (miles) }\end{array}$} & \multirow[t]{2}{*}{ Relao16n } & & & \\
\hline & & & & $I_{x}^{ \pm}$ & $\mathrm{L}_{x}$ & $8 L_{x}^{t} / L_{x}$ \\
\hline $\begin{array}{c}0-4 \\
20-24 \\
40-44 \\
60-64 \\
80-84\end{array}$ & $\begin{array}{rr}10 & 432 \\
6 & 539 \\
6 & 033 \\
3 & 710 \\
& 820\end{array}$ & $\begin{array}{rr}10 & 002 \\
6 & 794 \\
6 & 378 \\
4 & 099 \\
1 & 175\end{array}$ & $\begin{array}{l}1.0430 \\
0.9624 \\
0.9459 \\
0.9051 \\
0.6979\end{array}$ & $\begin{array}{rr}486 & 168 \\
476 & 823 \\
453 & 348 \\
345 & 754 \\
94 & 580\end{array}$ & $\begin{array}{ll}489 & 235 \\
484 & 298 \\
470 & 778 \\
410 & 024 \\
180 & 614\end{array}$ & $\begin{array}{l}1.0406 \\
1.0310 \\
1.0084 \\
0.8831 \\
0.5484\end{array}$ \\
\hline
\end{tabular}

En el caso de las edades mayores el modelo funciona bien, y muestra una relación de masculinidad que disminuye en forma sostenida. De hecho, el modelo alcanza un nivel más bajo que el observado. La diferencia entre la mortalidad masculina y femenina se ha acentuado 
en los últimos años, y esto explica el hecho de que el cociente observado sea más elevado que el estable; la discrepancia puede ser concebida como un rezago. Por ejemplo, el valor de $s L_{40}^{*} / L_{40}$ en 1944 1946 fue 0.9927 , contra 1.0094 que aparece en el cuadro 1-a, una diferencia pequeña, pero en la dirección correcta. La tendencia de $s L_{40}^{*} / L_{40}$ es en general a la baja durante 45 años:

$\begin{array}{ll}1919-1921 & 1.0310 \\ 1929-1931 & 1.0155 \\ 1939-1941 & 1.0131 \\ 1949-1951 & 1.0150 \\ 1959-1961 & 1.0118 \\ 1965 & 1.0084\end{array}$

Parte de las discrepancias del cuadro 1-a se deben al peso del elemento masculino en la inmigración durante la primera parte del siglo, que no ha sido tomada en cuenta en el modelo estable.

Sin embargo, el modelo da malos resultados en las edades de 20 y 40 . Esto se debe en parte a las fuerzas armadas fuera del país, que no han sido incluidas en la población observada, pero que sí están incluidas implícitamente en el modelo estable. La subenumeración de la población masculina joven del censo de 1960 es también en parte la causa, en vista de que se reflejaría en las estimaciones de 1965 que constituyen nuestras cifras "observadas".

El resultado simple, que no depende de $r$, fue posible al suponer que los dos sexos crecen a la misma tasa. En cualquier año, es probable que la $r$ masculina y la femenina, obtenidas respectivamente de información sobre la edad del padre y de la madre en el momento del nacimiento, difieran. Esto puede deberse a una declaración incorrecta sistemática de la edad $\mathrm{y}$, si no, debe desaparecer cuando se promedia un número suficiente de años.

Respecto a Austria, las dos guerras de este siglo fueron impuestas en los elementos estables y afectan seriamente las edades de 40 en adelante en 1965 (cuadro 2).

Cuadro 2

AUSTRIA: RELACIONES DE MASCULINIDAD OBSERVADAS Y ESTABLES, 1965

\begin{tabular}{|c|c|c|c|}
\hline$\underset{\text { oded }}{\text { arupos de }}$ & $\begin{array}{l}\text { Relaofbn do masoulinidad } \\
\text { observada }\end{array}$ & $\begin{array}{c}\text { Releoibn ogtable } \\
=L_{x}^{ \pm} / L_{x}\end{array}$ & $\begin{array}{c}\text { Cociente entre In } \\
\text { observade y li } \\
\text { ostable }\end{array}$ \\
\hline $\begin{array}{l}35-39 \\
40-44 \\
45-49 \\
50-54 \\
55-59 \\
60-64 \\
65-69 \\
70-74 \\
75-79 \\
80-84 \\
85 \mathrm{~J}+\end{array}$ & $\begin{array}{l}0.9459 \\
0.7293 \\
0.7317 \\
0.7617 \\
0.7841 \\
0.7984 \\
0.6956 \\
0.5947 \\
0.5524 \\
0.5340 \\
0.4783\end{array}$ & $\begin{array}{l}1.0116 \\
1.0034 \\
0.9925 \\
0.9772 \\
0.9487 \\
0.8939 \\
0.8133 \\
0.7183 \\
0.6196 \\
0.5330 \\
0.4234\end{array}$ & $\begin{array}{l}0.94 \\
0.73 \\
0.74 \\
0.79 \\
0.83 \\
0.89 \\
0.86 \\
0.83 \\
0.89 \\
1.00 \\
1.13\end{array}$ \\
\hline
\end{tabular}


Nótese la pausa en la tendencia descendente de la relación observada entre las edades 50 y 60 , que representa el período de la guerra. La columna donde aparece el cociente entre la observada y la estable ayuda a poner de relieve los efectos de las guerras, mostrando valores mínimos alrededor de la edad de 40 y la de 70 .

Se esperaría que el número total de casados de ambos sexos fuera igual, pero de hecho no lo es. Las estimaciones oficiales de 1967 muestran (Statistical Abstract 1968, p. 32) 45.2 millones de hombres casados y 46.2 millones de mujeres. Por más modernos que seamos, el. matrimonio es todavía una exigencia por parte de la mujer, una confesión por parte del hombre, y evidentemente se hacen a los enumeradores censales cerca de un millón de exigencias más que de confesiones.

Las disparidades más fuertes en cifras se encuentran entre los viudos y las viudas. En las edades 55-64, por ejemplo, se reportaron 2.0 millones de viudas y sólo 0.4 millones de viudos en los Estados Unidos. Debido a las diferencias de mortalidad, los hombres son menos numerosos que las mujeres, digamos en 0.8 millones, en el intervalo de edad. Esto explica cerca de la mitad del exceso de 1.6 millones de viudas sobre viudos, y que existiría si los maridos y las esposas fueran de la misma edad. El 0.8 restante es atribuible al hecho de que las mujeres se aparean con hombres de mayor edad que ellas.

\section{DIFERENCTAS EN LA MORTALIDAD POR SEXOS}

Cuando menos en las dos últimas generaciones, la mortalidad femenina ha sido menor que la masculina. En las tablas de vida de 1920 de los Estados Unidos la esperanza de vida al nacer fue 54.7 años en el caso de los hombres y 56.4 en el de las mujeres. La diferencia aumentó a un ritmo acelerado y para 1967 la de los hombres era 67.0 y las de las mujeres 74.3, una diferencia de más de 7 años, y al parecer tođavía creciente. Ésta fue la causa de la disminución de las relaciones de masculinidad hacia el extremo superior de la vida observada en los ejemplos de la sección anterior.

Una parte de la diferencia es el exceso muy conocido de la mortalidad infantil masculina, pero la esperanza de vida a la edad 1 está totalmente libre de esta componente, y aquí encontramos 67.7 entre los hombres y 74.7 para las mujeres, una diferencia aún de 7 años.

Aun cuando se eliminen todas las edades hasta la de 50 , quedan todavía las mujeres con 5.5 años de ventaja. Después de esto la diferencia desciende, hasta ser de sólo 2.65 en la edad de 70 . Este exceso en las edades mayores es un fenómeno más nuevo que el exceso global; en 1920 la $\stackrel{0}{e}_{70} *$ femenina fue solamente 0.3 años superior a la masculina.

¿En qué grado se presenta el aumento de la esperanza de vida femenina en relación a la masculina, en todas las edades pero en especial en las mayores, en los países avanzados? Ciertamente se presenta en Inglaterra y Gales, donde la ventaja femenina fue de dos y medio años en 1861, ventaja que había aumentado a 6.2 años para 1966-1968. La

* El autor denota con este símbolo la esperanza de vida al nacer. [T.] 
diferencia en Francia es la más alta: calculamos 68.0 en 1967 para los hombres y 75.5 para las mujeres. Para Europa tomada globalmente encontramos 5.1 años en 1960, y para los países del Mercado Común 5.5 años. En 19.65 las diferencias en Europa se habían elevado a 5.6 años, con esperanzas masculinas de 67.7 y 73.3 para las femeninas; las diferencias en el Mercado Común fueron mayores de 6 años. El Canadá y Australia no se quedan atrás.

La mortalidad de los países subdesarrollados ha llegado a parecerse, en cuanto a su nivel, a la de los países desarrollados. Aparte de Africa Tropical, que tiene la mortalidad del siglo xvIII, los países del tercer mundo se encuentran en el lugar de Europa y América de los años veinte. Un aspecto en el que tienen avances en particular es en las diferencias entre sexos: son comunes las diferencias de 4,5 y 6 años.

Estos resultados basados en la esperanza de vida son apenas un aspecto de la diferencia entre la mortalidad global de los sexos. Una de sus peculiaridades es una ponderación implícita de las distintas edades según la distribución estacionaria por edades, y ésta difiere entre hombres y mujeres. También la distribución estacionaria por edades tanto para hombres como para mujeres está mucho más ponderada hacia las edades mayores - cuando menos cuando la población crece. En cierto sentido las esperanzas dicen la historia de los individuos; nos interesan las poblaciones, y para ellas la historia resulta ser muy diferente.

Una forma de análisis es en categorías individuales por sexo y edad. Respecto a los Estados Unidos en 1967 encontramos las tasas por edades para hombres y mujeres y calculamos la relación entre

\section{Cuadro 3}

Estados UNidos Y ElRoPa *: RELACIÓN ENTRE LA MORTALIDAD MASCULINa Y LA FEMENINA

\begin{tabular}{lcc}
\hline Eded & $\begin{array}{c}\text { Estados Unidos } \\
1967\end{array}$ & $\begin{array}{c}\text { Europa } \\
1965\end{array}$ \\
\hline 0 & 1.30 & 1.25 \\
1 & 1.25 & 1.15 \\
5 & 1.38 & 1.05 \\
10 & 1.72 & 1.15 \\
15 & 2.51 & 2.18 \\
20 & 2.80 & 2.33 \\
25 & 2.26 & 1.92 \\
30 & 1.81 & 1.73 \\
35 & 1.68 & 1.64 \\
40 & 1.72 & 1.57 \\
45 & 1.79 & 1.62 \\
50 & 1.95 & 1.78 \\
55 & 2.07 & 1.95 \\
60 & 2.10 & 1.95 \\
65 & 1.91 & 1.57 \\
70 & 1.82 & 1.39 \\
75 & 1.56 & 1.27 \\
80 & 1.33 & 1.13 \\
$85+$ & 1.08 & \\
\hline
\end{tabular}

* Al occidente de la URSS, omitiendo solamente Irlanda del Norte. La población incluida sumó 442 millones de habitantes. 


\section{Gráfica 2}

Estados Unidos: Poblactón masculina y femenina Por edades, 1967

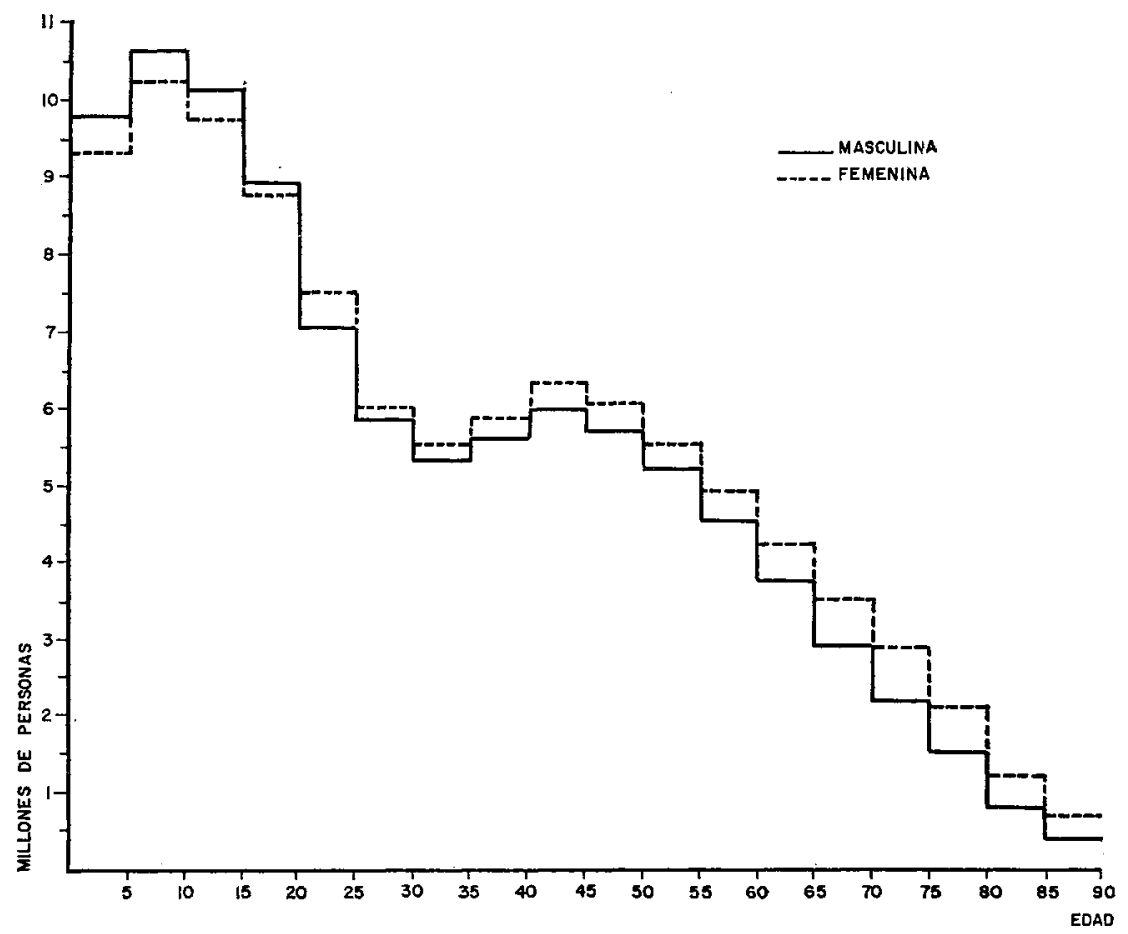

una y la otra. Se obtienen cifras similares en el caso de nuestra consolidación de Europa al occidente de la URSS; esta agrupación, con una población total de 442 millones, incluyó todos los países excepto Irlanda del Norte.

\section{Cuadro 4}

Estados Unidos: Defunciones POR EDAD Y SEXo, POR dos causas, 1967

\begin{tabular}{|c|c|c|c|c|}
\hline \multirow[t]{2}{*}{ Edad } & \multicolumn{2}{|c|}{$\begin{array}{l}\text { Accidentes en vehioulos } \\
\text { de motor } \mathrm{E} 810-\mathrm{E} 835\end{array}$} & \multicolumn{2}{|c|}{$\begin{array}{l}\text { Arteriosclerosis } y \text { enfermed ader } \\
\text { oardfacas degenerativas }\end{array}$} \\
\hline & Hombres & Ku jeres & Hombres & Mu jeres \\
\hline \multirow[t]{2}{*}{$\begin{array}{l}-15 \\
15-24 \\
25-34 \\
35-44 \\
45-54 \\
55-64 \\
65-74 \\
75 \text { y + } \\
\text { Total de la } \\
\text { edad declarada }\end{array}$} & $\begin{array}{rr}3 & 669 \\
12 & 169 \\
5 & 689 \\
4 & 257 \\
4 & 144 \\
3 & 467 \\
2 & 702 \\
2 & 017\end{array}$ & $\begin{array}{ll}2 & 243 \\
3 & 477 \\
1 & 528 \\
1 & 513 \\
1 & 675 \\
1 & 616 \\
1 & 583 \\
1 & 152\end{array}$ & $\begin{array}{r}28 \\
113 \\
1058 \\
10284 \\
36923 \\
74136 \\
100689 \\
121786\end{array}$ & $\begin{array}{r}15 \\
57 \\
362 \\
2 \quad 229 \\
869 \\
24748 \\
59220 \\
132604\end{array}$ \\
\hline & $38 \quad 114$ & 14787 & 345067 & 227933 \\
\hline
\end{tabular}

Fuente: Vital Statistics of the United States, 1967. Mortality, Vol. 2. 
Los cocientes de tasas de mortalidad muestran un patrón característico. Empiezan con el exceso clásico de 25 o $30 \%$ de mortalidad infantil masculina, luego disminuyen ligeramente, luego se elevan a un máximo de cerca de 2.5 a 1 en la edad 20-24, disminuyen de nuevo a un nivel bajo alrededor de las edades entre 35 y 45 , de allí se elevan de nuevo hasta cerca de 2 a la edad de 60 ; finalmente descienden hasta alcanzar la igualdad a las edades de 85 y más. El patrón de tres prominencias (una menor a la edad 0 y dos grandes alrededor de las edades de 20 y 60) y tres depresiones (una menor alrededor de los 5 años y dos grandes alrededor de los 40 y los 85) se repite una y otra vez en países individuales en los tiempos modernos (véanse la gráfica 2 y el cuadro 3 ).

La curva fue mucho menos aguda en la Suecia del siglo xvint, y hoy en día es menos aguda en países como Madagascar. Es menos aguda - aunque esencialmente de la misma forma- en la población no blanca de los Estados Unidos de la actualidad. En los lugares y en las fechas de mortalidad fuerte, los hombres y las mujeres tienden a sufrir por parejo el fenómeno.

En épocas de elevada mortalidad rara vez se dispone de la distribución por causas. Uno puede especular que las enfermedades infecciosas son menos discriminatorias en relación con el sexo, que las causas tan importantes de la mortalidad contemporánea en los países avanzados como los accidentes y las enfermedades del corazón. El mayor exceso masculino a principios de los años veinte se relaciona probablemente con lo anterior, y en las edades de los sesentas con lo segundo. Nótese que ambas prominencias son más elevadas en Estados Unidos que en Europa.

En el cuadro 4 aparece una sugerencia para el análisis por causas en los Estados Unidos. Asombra la prominencia de los accidentes de hombres en las edades 15-24, tanto en relación con otras edades como con las mujeres. Es también notable la mortalidad masculina mucho mayor debida a enfermedades cardíacas, cuando menos hasta los 70 años.

La siguiente cuestión es la de cómo sumar los relativos del cuadro 3. Ciertamente debiéramos poder decir que la mortalidad masculina está promediada globalmente para todas las edades, y que es tanto por ciento mayor que la mortalidad femenina.

Supóngase que ponderamos las distintas edades de acuerdo con el número de individuos de la población. Con ponderaciones masculinas la fórmula puede expresarse:

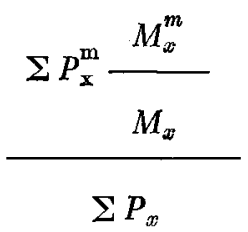

donde $P_{x}$ es la población masculina de las edades $x$ a $x+4$ (como es usual, trataremos grupos quinquenales) y $M_{x}$ es la mortalidad masculina del mismo grupo de edad, mientras que las características 
femeninas se denotarán con $f$ en lugar de $m$. En los Estados Unidos, en 1967, el cociente entre la mortalidad masculina y la femenina resulta ser 1.871 con base en la medida anterior. Con la misma fórmula, pero ponderando con la población femenina, encontramos un cociente de 1.874 .

Esto es análogo a comparar los precios de dos ciudades promediando sus precios relativos - los cocientes del precio de una ciudad sobre los de la otra, artículo por artículo, ponderados según la importancia de los bienes de acuerdo con la estructura del gasto. Pero también puede hacerse una comparación diferente - expresada en las discusiones de índices de precios como el costo de llenar una canasta de bienes con una cierta lista de artículos de las dos ciudades. Esto recibe el nombre de índice agregativo, $y$ es un candidato para nuestras consideraciones presentes.

En nuestros términos hacemos la pregunta de cuántas mujeres morirían en la misma población si las mujeres tuvieran la distribución por edades actual, representada por $P_{x}^{f}$, pero estuvieran sujetas, edad por edad, a las mismas tasas de mortalidad. EI número de defunciones sería $P_{x}^{f} M_{x}^{m}$, y podemos comparar éste con el número de defunciones realmente acontecidas, $P_{x}^{f} M_{x}$, para formar el índice:

$$
\frac{\Sigma P_{x}^{f} M_{x}^{m}}{\sum P_{x}^{f} M_{x}^{f}}
$$

Este índice agregativo nos da como resultado 1.61, mucho menor que el promedio de relativos. Al intentar ver el porqué de la diferencia, podemos reescribirlo multiplicando y dividiendo el numerador por $\boldsymbol{M}_{x}^{f}$, de modo que la expresión queda en la forma de un promedio ponderado de relativos:

$$
\frac{\sum P_{x}^{f} M_{x}^{f} \frac{M_{x}^{m}}{M_{x}^{f}}}{\Sigma P_{x}^{f} M_{x}^{f}}
$$

Éste es el mismo que (1) excepto que la ponderación no es ahora la población sino las defunciones en las distintas edades. Al ponderar con defunciones femeninas este índice da un $27 \%$ de exceso de la mortalidad masculina sobre la femenina en los Estados Unidos en 1967, y al ponderar con las defunciones masculinas el exceso es de $61 \%$.

El perfil de ponderaciones de población empieza con valores elevados y disminuye gradualmente hacia el extremo superior de la vida. El perfil de defunciones empieza con valores elevados, luego disminuye a niveles muy bajos y luego se eleva a sus valores mayores en los últimos grupos de edad. De este modo, cerca del $60 \%$ de las defun- 
ciones femeninas de los Estados Unidos se presentaron entre mujeres mayores de 70 años, donde el cociente $M_{x}^{m} / M_{x}^{f}$ desciende de 1.82 en las edades $70-74$ a 1.08 en las de 85 y más, y este último es el mayor grupo de defunciones. Y el $17 \%$ de las defunciones femeninas están en este grupo terminal, pero sólo $9 \%$ de las defunciones masculinas.

Al elegir entre ponderar con población o con defunciones debemos, entonces, decidir qué queremos: $\dot{c}$ las ponderaciones que ponen mucho énfasis en las edades de 10 a 30 , donde el cociente es mayor que 2.0 en su mayor parte, o la que pone énfasis en las edades superiores, donde el cociente disminuye gradualmente a la unidad? Una manera de dar respuesta a esto es pensando acerca del efecto de un cambio de una defunción. Si ponderamos con la población, entonces un cambio de una defunción altera más el índice en las edades 10-30, donde hay pocas defunciones, en comparación con lo que sucede en las edades mayores de 70 años. Por otra parte, en el índice agregativo el efecto de un cambio de una defunción es más o menos el mismo en todas las edades.

Este argumento favorece el uso de las defunciones como ponderaciones. Pero la ponderación con defunciones masculinas da un exceso masculino de $61 \%$, y con las femeninas de $27 \%$. Este intervalo parece lo más que podemos aproximarnos para hacer una afirmación sobre el exceso de mortalidad masculina sobre la femenina en los Estados Unidos.

\section{Número ESPERADO DE PARENTESCOS SANGUÍNEOS}

Una parte del significado del conjunto de tasas de mortalidad por edad y sexo es el número de hermanos y hermanas, sobrinas, tîas y otros parientes que se espera tenga una persona. Dista mucho de ser obvio cómo el número de tías maternas vivas que puede esperarse tenga un joven de 15 años pueda ser derivado de las simples tasas de mortalidad por edad y sexo, y se requiere una cadena de razonamientos para derivar las fórmulas. Leo Goodman y el autor proporcionaron derivaciones con cierto detalle en otro trabajo; para el propósito presente de interpretación de la edad y el sexo una discusión más breve será suficiente.

Aunque todo parte de un conjunto de tasas de natalidad y mortalidad por edades, digamos femeninas, a partir de éstas puede construirse una tabla de vida, de modo que de hecho tenemos:

a) La probabilidad de que una mujer recién nacida sobreviva a la edad $x$, denotada por $\ell_{r}$.

b) La probabilidad de que una mujer de edad $x$ tenga un hijo en los siguientes $d x$ años, denotada por $m_{x} d x$. Se supone que ésta es positiva en el intervalo comprendido entre $\alpha$ y $\beta$, y las edades entre $\alpha$ y $\beta$ son los límites de la vida reproductiva de los individuos. Suponemos que estas tasas son fijas -el objeto es encontrar qué cantidad de hermanas, sobrinas, etc., están implicadas por un régimen de mortalidad y fecundidad. 
Si las tasas específicas por edades son constantes y conocemos las $\ell_{x}$ y las $m_{x}$, podemos entonces inferir seis cosas:

1) La distribución por edades de la población. La proporción de individuos entre las edades $x$ y $x+d x$ debe ser $b e^{-r x} \ell_{x} d x$, donde $b=1 / \int e^{-r x} \ell_{x} d x$ es la tasa de natalidad global, y $r$ es la tasa de crecimiento de la población; éste es el resultado debido a Euler que examinamos previamente.

2) La distribución por edades de las madres de hijos nacidos en cualquier momento dado. Si el número de mujeres entre las edades $x$ y $x+d x$ es $b e^{-r x} R_{x} d x$ y la tasa de natalidad de estas mujeres es $m_{x}$, entonces el número de madres por habitante entre las edades $x$ y $x+d x$ debe ser $b e^{-r x} g_{x} m_{x} d x$; el número de madres entre las edades $x$ y $x+d x$ por nacimiento debe ser la misma cantidad, pero sin el factor $b$; en breve, $f(x) d x=$ $e^{-r x} \alpha_{x} m_{x} d x$ es la probabilidad de que la madre de un niño o una persona mayor escogida al azar tenga la edad $x$.

3) La tasa de crecimiento $r$. Mientras que la tasa $r$ a la cual tiende a crecer la población se deriva de ordinario de diferentes consideraciones, podemos ver que el número de madres (sumando todas las edades) por cada nacimiento debe ser igual a 1 , es decir:

$$
\int_{\alpha}^{\beta} e^{-r x} \ell_{x} m_{x} d x=1
$$

La ecuación es suficiente para determinar $r$ cuando se conocen $\ell_{x}$ y $m_{x}$.

4) El número esperado de hijos alguna vez nacidos de mujeres de edad $a$. Éste debe ser:

$$
\int_{\alpha}^{a} m_{x} d x, a>\alpha>0
$$

No se requiere factor alguno de supervivencia de la madre -el hecho de que tenga edad $a$ implica que ha vivido todos los años a precedentes.

5) Sus hijos, sin embargo, pueden no haber sobrevivido, y el número de ellos actualmente vivos es:

$$
\int_{\alpha}^{a} m_{x} \ell_{a-x} d x, a>\alpha>0
$$

6) El número futuro de hijos de una mujer que actualmente tiene edad a durante su vida reproductiva es:

$$
\int_{a}^{\beta} \frac{\ell_{x}}{\ell_{a}} m_{x} d x, \beta>a>0
$$


Aquí necesitamos el factor $\ell_{\infty} / R_{a}$ para proporcionar la supervivencia de la mujer en cada edad de posible alumbramiento.

De 2) podemos derivar la probabilidad de que la mujer de edad actual $a$ tenga una madre viva. En cuanto a la probabilidad de que su madre haya tenido la edad $x$ al nacimiento $a$ años antes, ésta es $f(x) d x$; si ella tenía la edad $x$, entonces la probabilidad de que actualmente viva es $8_{a+x} / \ell_{a}$; multiplicando estas dos probabilidades, y promediando sobre todas las $x$, tenemos:

$$
M_{1}(a)=\int_{\alpha}^{\beta} \frac{\ell_{x+a}}{\ell_{a}} f(x) d x
$$

que es una función de a solamente. Ello es un poco más general que lo expresado antes; la muchacha nacida hace a años no necesita haber sobrevivido, y $M_{1}(a)$ sería aún la probabilidad de que su madre viviera $a$ años después de haber dado a luz. Pero es esencial que el nacimiento de hace $a$ años sea seleccionado al azar.

¿Cuál es la probabilidad de que la muchacha de edad a tenga abuela viva? Supóngase que la muchacha de edad a nació cuando su madre tenía edad entre $x$ y $x+d x$, de lo cual la probabilidad es $f(x) d x$. Entonces la cuestión es la de si su abuela vivió $x+a$ años después de haber dado a luz, y según el párrafo anterior esto debe ser $M_{1}(x+a)$. La probabilidad de que la abuela sobreviva y de que la madre dé a luz a la edad $x$ es el producto $M_{1}(x+a) f(x) d x$. La probabilidad total de que la madre sobreviva es la integral

$$
M_{2}(x)=\int_{\alpha}^{\beta} M_{1}(x+a) f(x) d x
$$

De igual manera, para el progenitor de la generación $i$ precedente, de una muchacha que actualmente tiene la edad $a$, la probabilidad de supervivencia al presente es:

$$
M_{i}(a)=\int_{\alpha}^{\beta} M_{i-1}(x+a) f(x) d x
$$

Ésta es la probabilidad de que una persona de edad a escogida al azar tenga una abuela viva de orden $i-2$ por la línea materna.

El modelo unisexual utilizado hasta aquí se aplica igualmente a los hombres. El cuadro 5 muestra las probabilidades de que las progenitoras femeninas estén vivas, y la de los progenitores masculinos, con base en las tasas de natalidad y mortalidad por edades de los Estados Unidos en 1967. Dice, por ejemplo, que la probabilidad de que una persona de 20 años de edad tenga madre viva en una población con las tasas por edades de los Estados Unidos en 1967 es 0.9594. Por lo tanto la probabilidad de que pierda a su madre es 1-0.9594 o 


\section{Cuadro 5}

Probabilidad de vida de la madre, de la abuela, ..., DEL Padre, Del abuelo, ..., SEGÚN LAS TASAS DE NATALIDAD DE LOS EsTadOS UNIDOS DE 1967

Las probabilidades se refieren a personas tomadas al azar

\begin{tabular}{|c|c|c|c|c|}
\hline $\begin{array}{c}\text { Edad } \\
\text { a }\end{array}$ & Madre & Abnela & B1sabue1a & Tatarabuela \\
\hline \multirow[t]{2}{*}{$\begin{array}{l}0 \\
5 \\
10 \\
15 \\
20 \\
25 \\
30 \\
35 \\
40 \\
45 \\
50 \\
55 \\
60\end{array}$} & $\begin{array}{r}1.0000 \\
.9946 \\
.9870 \\
.9758 \\
.9594 \\
.9355 \\
.9012 \\
.8525 \\
.7842 \\
.6927 \\
.5764 \\
.4388 \\
.2971\end{array}$ & $\begin{array}{l}.9359 \\
.9023 \\
.8550 \\
.7904 \\
.7054 \\
.5995 \\
.4760 \\
.3440 \\
.2177 \\
.1117 \\
.0391 \\
.0068 \\
.0002\end{array}$ & $\begin{array}{l}.6208 \\
.5079 \\
.3865 \\
.2671 \\
.1622 \\
.0821 \\
.0317 \\
.0081 \\
.0011 \\
.0000 \\
.0000 \\
.0000 \\
.0000\end{array}$ & $\begin{array}{l}.1227 \\
.0620 \\
.0254 \\
.0078 \\
.0016 \\
.0002 \\
.0000 \\
.0000 \\
.0000 \\
.0000 \\
.0000 \\
.0000 \\
.0000\end{array}$ \\
\hline & Eadro & Abaele & Bisabuele & Tatarabuele \\
\hline \multirow[t]{2}{*}{$\begin{array}{c}0 \\
5 \\
10 \\
15 \\
20 \\
25 \\
30 \\
35 \\
40 \\
45 \\
50 \\
55 \\
60\end{array}$} & $\begin{array}{r}1.0000 \\
.9870 \\
.9697 \\
.9449 \\
.9084 \\
.8559 \\
.7833 \\
.6890 \\
.5747 \\
.4475 \\
.3195 \\
.2046 \\
.1143\end{array}$ & $\begin{array}{l}.8236 \\
.7467 \\
.6516 \\
.5416 \\
.4235 \\
.3070 \\
.2026 \\
.1184 \\
.0578 \\
.0204 \\
.0036 \\
.0002 \\
.0000\end{array}$ & $\begin{array}{l}.2954 \\
.1999 \\
.1221 \\
.0653 \\
.0289 \\
.0095 \\
.0020 \\
.0002 \\
.0000 \\
.0000 \\
.0000 \\
.0000 \\
.0000\end{array}$ & $\begin{array}{l}.0145 \\
.0046 \\
.0010 \\
.0001 \\
.0000 \\
.0000 \\
.0000 \\
.0000 \\
.0000 \\
.0000 \\
.0000 \\
.0000 \\
.0000\end{array}$ \\
\hline & \multicolumn{4}{|c|}{$\begin{array}{l}\text { Edader del padro al nacimiento de los hijos pero oon mortalidad } \\
\text { femenina on todas las generacionea }\end{array}$} \\
\hline $\begin{array}{r}0 \\
5 \\
10 \\
15 \\
20 \\
25 \\
30 \\
35 \\
40 \\
45 \\
50 \\
55 \\
60\end{array}$ & $\begin{array}{r}1.0000 \\
.9933 \\
.9836 \\
.9695 \\
.9488 \\
.9190 \\
.8766 \\
.8178 \\
.7374 \\
.6330 \\
.5070 \\
.3672 \\
.2308\end{array}$ & $\begin{array}{l}.8973 \\
.8481 \\
.7821 \\
.6967 \\
.5919 \\
.4714 \\
.3439 \\
.2214 \\
.1177 \\
.0443 \\
.0081 \\
.0005 \\
.0000\end{array}$ & $\begin{array}{l}.4423 \\
.3254 \\
.2161 \\
.1247 \\
.0588 \\
.0204 \\
.0044 \\
.0005 \\
.0000 \\
.0000 \\
.0000 \\
.0000 \\
.0000\end{array}$ & $\begin{array}{l}.0295 \\
.0098 \\
.0023 \\
.0003 \\
.0000 \\
.0000 \\
.0000 \\
.0000 \\
.0000 \\
.0000 \\
.0000 \\
.0000 \\
.0000\end{array}$ \\
\hline
\end{tabular}

$4.06 \%$. Esto ha disminuido fuertemente desde 1920, cuando Lotka (1931) hizo tales cálculos por primera vez y encontró que el $7 \%$ de los niños de edad 20 serían huérfanos maternos según las tasas de natalidad y mortalidad de la época.

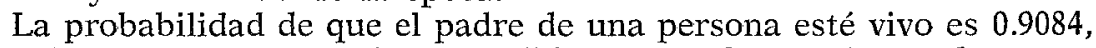
y la de que una persona haya perdido a su padre es el complemento, 0.0916. Según estas tasas habría cerca de 2.25 veces más huérfanos de padre que de madre. Las probabilidades se aplican sin cambio a las madres de muchachos y los padres de muchachas.

$¿$ Cuál es la probabilidad de que un niño haya perdido a su madre y a su padre? Esto resulta ser un problema mucho más difícil. Uno 
podría dar como respuesta el producto $0.0916 \times 0.0406$, para obtener 0.0036 que es cerca de uno en 300 . Esto es probablemente muy bajo en la medida en que hombres saludables tienden a casarse con mujeres saludables. La mortalidad de los padres difícilmente es independiente de la mortalidad de las madres. Hasta aquí no hemos hecho supuesto alguno acerca de la distribución de los nacimientos o las defunciones entre los individuos, y mucho menos hemos supuesto independencia entre las de un individuo y las de otro.

Las diferencias entre los sexos pueden ser estudiadas en las edades mayores y para ancestros más viejos mediante el cuadro 5 basado en información de Estados Unidos de 1967. La probabilidad de que la madre esté viva en una persona de 50 años de edad es 0.576 , mien. tras que la probabilidad de que el padre viva es sólo 0.319. Hemos visto que en muchas edades la mortalidad masculina es el doble de la femenina, y la diferencia se acentúa cuando los padres son alrededor de tres años mayores.

A medida que se observan generaciones más viejas el efecto se multiplica. De este modo la probabilidad de que una persona de 50 años tenga abuela es 0.039 , y la de tener abuelo es menos de la décima parte de la anterior, 0.0036 . La probabilidad de que un recién nacido tenga bisabuela es 0.123 , pero la de que tenga bisabuelo es sólo 0.0145 , cerca de un octavo de la anterior.

La mayor presencia de ancestros por la línea femenina se debe tanto a la mortalidad femenina más favorable como al hecho de que son más jóvenes que los maridos; pero en los resultados del cuadro 5 en ocasiones uno de estos factores tiene el efecto mayor, y en ocasiones lo tiene el otro. El asunto se investiga fácilmente mediante un dispositivo que utilizamos antes, aplicado en este caso a ver lo que sería la probabilidad de supervivencia de los hombres si retuvieran su mortalidad pero si fueran de la misma edad que sus esposas. La tercera sección del cuadro 5 muestra probabilidades mezcladas sobre esta base.

El resultado de asignar a los hombres la mortalidad de las mujeres equivale a cubrir tres cuartas partes de la diferencia entre la probabilidad de que una persona de 50 años tenga madre y padre -se convierte a 0.507 . Por otra parte, ello no cambia sustancialmente la probabilidad de que un recién nacido tenga bisabuelo, elevándola a sólo 0.0295. La edad al nacimiento actúa en cada generación, y de este modo su efecto es multiplicado en las relaciones que abarcan varias generaciones. La edad del hombre al hacerse padre opera cuatro veces al hacer el cálculo para los tatarabuelos, y sólo una vez en el cálculo de las madres y los padres. Para estos últimos la mayor mortalidad de los hombres es el factor más importante; las diferencias entre las madres y los padres vivos se observan en el cuadro 5 , y son más cercanas entre la primera y la tercera sección, mientras que las de los tatarabuelos son más cercanas entre las secciones segunda y tercera

\section{EL NÚMERO DE PRIMOS}

Para ilustrar en un caso más complejo qué tan fácilmente se deriva el número esperado de parientes a partir de las proposiciones gene- 
rales que acabamos de presentar, derivemos una expresión para los primos hermanos de madre, es decir primos cuyas madres son hermanas. Empezamos con una muchacha de edad $a$, e inquirimos acerca de sus primas hijas de las hermanas mayores de su madre.

La probabilidad de que su madre tenga edad entre $x$ y $x+d x$ al nacimiento de su hija es $f(x) d x$, y la de que su abuela haya tenido $y$ años al nacimiento de su madre es $f(y) d y$. La probabilidad de que su abuela tuviera una hija mayor a la edad $z$, donde $z<y$, es $m_{z} d z$. El número futuro de hijas de esta hermana mayor de su madre sería $\ell_{w} m_{w} d w$, supuestos a partir de $\alpha$ hasta el presente, que equivale a decir $a+x+y-z$ utilizando el artificio de contar el tiempo a lo largo de las edades de las distintas personas involucradas. Para encontrar la respuesta completa necesitamos sólo integrar sobre todas las posibles edades $x$ de la madre, las edades $y$ de la abuela, y $z$ de la hermana mayor de la madre, para obtener

$$
\int_{\alpha}^{\beta}\left(\int_{\alpha}^{\beta}\left(\int_{\alpha}^{y}\left(\int_{\alpha}^{a+x+y-z} \ell_{w} m_{w} d w\right) m_{z} d z\right) f(y) d y\right) f(x) d x
$$

Respecto de los primos cuya madre es menor que la madre de la muchacha de edad a sólo necesitamos reemplazar la integral sobre $z$. Tenemos que tomar en cuenta la supervivencia de la abuela a esa edad $z$, y para hacerlo reemplazamos $m_{z}$ de la expresión anterior por $\left(g_{z} / \boldsymbol{g}_{y}\right) m_{z}$. Además, los límites de integración correspondientes tienen que ser modificados; en lugar de hacerlo de $\alpha$ a $y$ lo hacemos de $y$ a $a+x+y$. Por lo tanto tenemos:

$$
\int_{\alpha}^{\beta}\left(\int_{\alpha}^{\beta}\left(\int_{y}^{a+x+y}\left(\int_{\alpha}^{a+x+y-z} \varepsilon_{w} m_{w} d w\right) \frac{\ell_{z}}{\ell_{y}} m_{z} d z\right) f(y) d y\right) f(x) d x
$$

que se refiere a los primos cuya madre es hermana menor de la muchacha de edad $a$.

La suma de las dos integrales daría el número esperado de primos relacionados a través de hermanas de la madre de la persona. En cuanto al número de éstos que aún viven sólo tenemos que introducir en la integral central de cada uno el factor $\ell_{a+x+y-z-w^{*}}$

Estos cálculos de Leo Goodman y el autor sobre valores esperados se presentan aquí como ejemplos del alcance de las inferencias que pueden hacerse sobre una población de la que sólo se conocen sus tasas específicas de natalidad y mortalidad por edades. Con sólo un elemento más - la proporción de mujeres casadas en las distintas edades - se puede calcular el tamaño promedio del hogar. En vista de que la distribución por edades de las madres al nacimiento de sus hijos no es muy diferente a la distribución correspondiente al nacimiento de sus hijas, las mismas expresiones servirían para el cálculo de los parientes de un muchacho de edad $a$, aunque también tendríamos que tomarlo al azar. Más aún, los parientes masculinos por la línea masculina se obtienen simplemente aplicando la tabla de vida 
masculina y las edades de los padres al nacimiento de sus hijos. Respecto de las líneas cruzadas - tíos por parte de la madre, o primos cruzados - se requiere tener cuidado en aplicar las fórmulas al utilizar las tablas de vida y las tasas de fecundidad apropiadas en cada ocasión.

Mi interés está en ver cuáles son las implicaciones de un régimen fijo. Si uno desea encontrar las implicaciones de un régimen cambiante, tal como ocurre en la práctica, se tendría que reemplazar en cada una de las fórmulas anteriores cada probabilidad por la prevaleciente en ese tiempo. Por ejemplo, se requerirían tablas de vida de cohorte y tasas de fertilidad de cohortes. En cuanto a las varianzas y los momentos de orden superior de dichos números, se requeriría mayor información, o supuestos adicionales: se necesitaría conocer evidentemente algo acerca de la probabilidad de que una mujer tenga un hijo a la edad $y$ dado que tuvo uno a la edad $x$, lo cual es una restricción que no afecta los valores globales esperados.

El modelo estable explica muchos fenómenos, de los cuales hemos examinado algunos. Pero no explica todo. En poblaciones modernas como la de los Estados Unidos, las variaciones considerables de las tasas de natalidad dejan su huella en la distribución por edades, y la fórmula de Euler ya no opera. Necesitamos una técnica más general para el análisis de tales casos: la teoría de matrices.

\section{INESTABILIDAD DE LA POBLACIÓN}

La técnica matricial para el análisis de la población debida a Leslie (1945) nos permite construir un operador que proyecta la distribución por edades hacia el futuro. Nos permite también descomponer la proyección en una componente exponencial $\mathrm{y}$ ondulaciones de diversas longitudes y amplitudes. En los Estados Unidos los ecos de la elevada natalidad de los años cincuenta se presentarían en los sesenta y los noventa si las tasas por edades permanecen constantes, y estos ecos pueden ser estimados con el método de Leslie. La inestabilidad produce también cambios en los cocientes de esposas y esposos potenciales, y requiere mayor estudio del mercado de matrimonios.

El mercado de matrimonios está constituido por los números de hombres y de mujeres de edades correspondientes. Qué son estas edades correspondientes en los Estados Unidos en 1967, lo sugiere la edad promedio del esposo al primer matrimonio, que es de 23.1 años, y la edad media de la esposa de 20.6, una diferencia de 2.5 años. (La edad promedio de los padres en la población estacionaria, en 1966, fue 29.33, y la de las madres 26.35, una diferencia de casi tres años.) Las cifras de las edades juveniles en Estados Unidos en 1966, fueron:

\begin{tabular}{ccc}
\hline & Hombres & Mujeres \\
\hline $10-14$ & 9861000 & 9542000 \\
$15-19$ & 8950000 & 8806000 \\
$20-24$ & 6625000 & 6981000 \\
$25-29$ & 5632000 & 5840000 \\
$30-34$ & 5326000 & 5527000 \\
\hline
\end{tabular}


La comparación entre las mujeres de un grupo de edad con los hombres del grupo cinco años mayor exagera la discrepancia, pero nos servirá como índice. En la información anterior podemos observar que la mayor desproporción en 1966 está entre las mujeres del grupo 15-19 y los hombres del grupo 20-24; a medida que éstos lleguen a la edad casadera, que es alrededor de estas fechas, el acortamiento de hombres será más serio que cinco años antes o cinco años después. En el Japón se observa el mismo fenómeno para 1966:

Hombres

3973000

4499000

5710000

4339000

4198000
Mujeres

3832000

4346000

5586000

4403000

4261000

El número de hombres entre 20 y 24 es mucho menor que el número de mujeres de 15-19, lo cual refleja un auge de bebés en los años cuarenta, pero de muy poca duración.

Este auge fue seguido por una disminución dramática de los nacimientos, y la situación inversa de los matrimonios se presenta entre los hombres del grupo 15-19 y las mujeres de 10-14. A medida que éstos alcancen la edad casadera en los años siguientes, el exceso presente de mujeres dará lugar a un acortamiento. La información sobre años posteriores nos diría en qué grado la disminución de la fecundidad a fines de los años sesenta (la tasa bruta de natalidad descendió de 18.6 en 1965 a 13.8 en 1966) se debe a la desproporción entre el número de hombres y mujeres en edades casaderas correspondientes. Este punto será importante para otros países que empiezan a hacer con éxito esfuerzos para controlar la fecundidad. La investigación al respecto es necesaria; no tenemos ni información ni teoría adecuadas acerca de la respuesta del matrimonio y la natalidad sobre el desproporcionamiento de los dos sexos. Éste es el problema más importante que no ha sido resuelto en la demografía teórica.

La posibilidad de que los padres puedan determinar el sexo de sus descendientes tendrá consecuencias demográficas y sociales importantes. En las sociedades donde se desean varones, y en las cuales los padres prosiguen teniendo hijos hasta que tienen el número deseado de varones, la tasa de natalidad disminuirá, y bajo el supuesto estático de preferencias fijas la magnitud de la disminución puede deri. varse inmediatamente a partir de consideraciones probabilísticas.

Sin embargo, es muy claro que las preferencias cambiarían antes de una generación como resultado de la escasez de mujeres. Se requiere de nuevo un modelo que incorporara el mercado de matrimonios; el número de varones jóvenes inicialmente mayor conduciría entre otras cosas a una disminución de la edad de las mujeres al matrimonio, y una elevación de la de los hombres. Se desarrollaría una situación análoga a la reducción de los matrimonios en los Estados 
KEYFITZ: SIGNIFICADO DEMOGRAFICO DE LA EDAD Y EL SEXO 191

Unidos. Esto resulta del auge de bebés de la posguerra, que constituyó un aumento simultáneo de hombres y mujeres. En consecuencia tenemos ahora una reducción de jóvenes dos o tres años mayores que las mujeres que alcanzan la edad casadera. El advenimiento del control del sexo donde se prefirieran varones implicaría una reducción mucho mayor de mujeres que de hombres. 